This item was submitted to Loughborough's Research Repository by the author.

Items in Figshare are protected by copyright, with all rights reserved, unless otherwise indicated.

\title{
Multilayer plasma patterns in atmospheric pressure glow discharges
}

PLEASE CITE THE PUBLISHED VERSION

PUBLISHER

(C) IEEE

VERSION

VoR (Version of Record)

LICENCE

CC BY-NC-ND 4.0

REPOSITORY RECORD

Yang, Y., J.J. Shi, J.E. Harry, John W. Proctor, Colin P. Garner, and Michael G. Kong. 2019. "Multilayer Plasma Patterns in Atmospheric Pressure Glow Discharges". figshare. https://hdl.handle.net/2134/5226. 
This item was submitted to Loughborough's Institutional Repository (https://dspace.lboro.ac.uk/) by the author and is made available under the following Creative Commons Licence conditions.

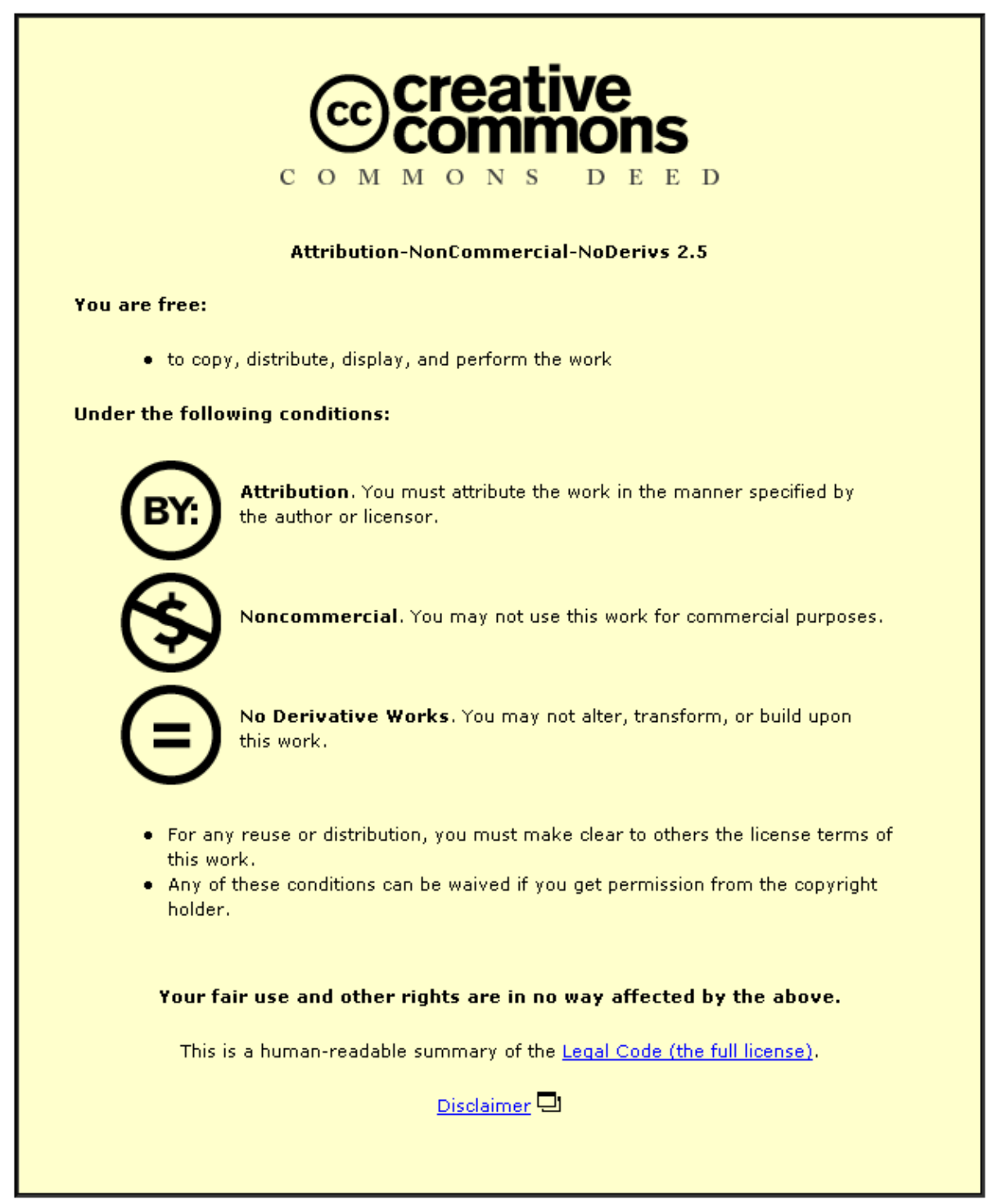

For the full text of this licence, please go to: http://creativecommons.org/licenses/by-nc-nd/2.5/ 


\title{
Multilayer Plasma Patterns in Atmospheric Pressure Glow Discharges
}

\author{
Y. Yang, J. J. Shi, J. E. Harry, J. Proctor, C. P. Garner, and M. G. Kong
}

\begin{abstract}
We report observation of self-organized multilayer plasma patterns formed along the length of an atmospheric pressure glow discharge generated over a wide frequency range from 10-100 kHz.
\end{abstract}

Index Terms-Atmospheric pressure glow discharges, double layers, self-organization, striation.

$\mathbf{S}^{\mathrm{H}}$ ELF-ORGANIZED formation of complex plasma patterns has been an intriguing subject of research with its impact on a variety of plasma systems [1]-[3]. In general, there are two broad groups of such complex plasma patterns: 1) self-organization of multiple plasma channels, and 2) self-organized multilayer patterns developed along the length of a single plasma column. In the first group, multiple plasma channels are formed typically between two parallel electrodes and their relative locations become locked with respect to each other. Such phenomena have been observed in laboratory generated discharges at different gas pressures, including atmospheric pressure [2], [3]. On the other hand, multilayer structures have been observed in both space plasmas and low-pressure glow discharges, usually in the form of a double-layer or multiple double-layers [4]. They are also known as striation [1]. To our knowledge, striation has so far not been reported in atmospheric pressure glow discharges (APGD). In this paper, we report observation of multilayer patterns in atmospheric helium glow plasmas.

Our plasma apparatus employs two pin electrodes to generate APGD in a helium flow of $5 \mathrm{slm}$ with a low-frequency power supply similar to that reported previously [6]. The pin electrodes are made of pure copper wires of $0.8 \mathrm{~mm}$ diameter and their separation distance is nominally $8 \mathrm{~mm}$. The pin electrodes are housed within a Perspex box to control environmental interference though the box is not air-tight. The sinusoidal highvoltage power supply of $1-10 \mathrm{kV}$ and $10-100 \mathrm{kHz}$ is connected to the plasma rig via a ballast resistor of $1 \mathrm{M} \Omega$. Electrical measurements were made via voltage and current probes. The rise time of the current probe is $2 \mathrm{~ns}$ and as such it should pick up nanosecond filamentary discharges. In general, the current traces were smooth and free of any nanosecond spikes. These

Manuscript received June 24, 2004; revised November 18, 2004. This work was supported by the U.K. Engineering and Physical Sciences Research Council (EPSRC).

Y. Yang, J. Proctor, and C. P. Garner are with School of Mechanical and Manufacturing Engineering, Loughborough University, Leicestershire LE11 3TU, U.K.

J. J. Shi, J. E. Harry, and M. G. Kong are with Department of Electronic and Electrical Engineering, Loughborough University, Leicestershire LE11 3TU,

U.K. (e-mail: m.g.kong@lboro.ac.uk).

Digital Object Identifier 10.1109/TPS.2005.845113 observations suggest that the generated atmospheric plasmas are glow discharges.

Fig. 1 shows the evolution of plasma structure from the usual glow mode to various striation modes. Fig. 1(a) shows a diffuse and homogenous glow discharge. The peak current density is $15 \mathrm{~mA} / \mathrm{cm}^{2}$. As the applied voltage is increased, a series of new modes of striated plasma column emerges, similar to multiple double layers observed in laboratory-produced low-pressure glow discharges and in ionosphere plasmas. In Fig. 1(b), there are four bright layers near each pin electrode. It is worth noting that these bright layers are of different thickness. From the pin electrode toward the center of the plasma column, the striated plasma layers become thinner and dimmer with thicker dark regions separating them. Near the pin electrodes, adjacent bright layers have a significant overlap in their optical emission yet these overlapped layers do not merge into each other. On the other hand, the two last bright layers nearest to the center of the plasma column are much more diffused and their optical emission has only slightly higher intensity than that of the central diffuse region. The striated pattern is very stable and stationary. The peak discharge density current for Fig. 1(b) is approximately $0.35 \mathrm{~A} / \mathrm{cm}^{2}$.

A small increase of the applied voltage evolves the striated pattern toward that of Fig. 1(c). The transition is sudden, similar to that from Fig. 1(a) to (b). Now there are five bright layers near each of the pin electrodes. The general characteristics remains that bright layers near the pin electrodes are brighter and thicker and they usually overlap each other. With the applied voltage increased further, the central homogenous is taken up by the striation pattern and the plasma column becomes fully striated, as shown in Fig. 1(d), where the generated plasma is seen with 12 bright layers distributed over its entire length. Again, the layers near the electrodes are brighter and thicker. The peak current density is now about $0.87 \mathrm{~A} / \mathrm{cm}^{2}$. For any two adjacent bright layers, either near the electrodes or near the central region, their separation distance does not appear to change significantly as the applied voltage increases.

So far, all striated APGD images are symmetric about the center of the plasma column. Under certain conditions, an asymmetric striation pattern can also be observed with the multilayer structure occurring near one electrode only as shown in Fig. 1(e). The overlapping among near-electrode bright layers is much more substantial than before and becomes a very bright and seemingly homogenous region. While not apparent from the image, this seemingly homogenous plasma region is indeed made from spatially static bright layers. At its end near the center of the plasma column, two-three distinct bright layers appear to sprout out into a diffuse and less bright region near 


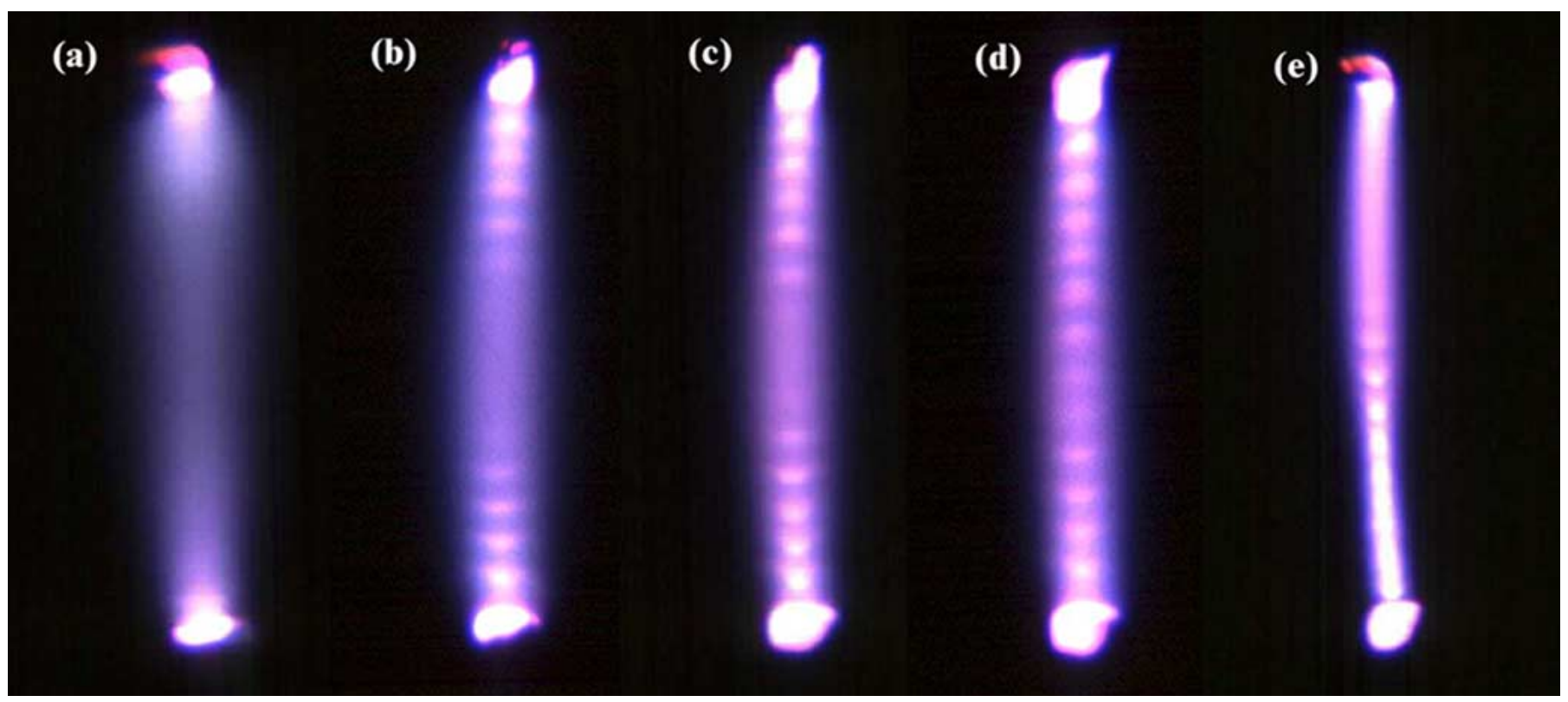

Fig. 1. Evolution of APGD images from the usual homogenous mode (a) to various striation modes including (b) a symmetric striated plasma mode with 4 bright layers near each of the two pin electrodes, (c) a similar striation mode now with 5 bright layers near each electrode, (d) the full striation mode, and (e) an asymmetric striation mode.

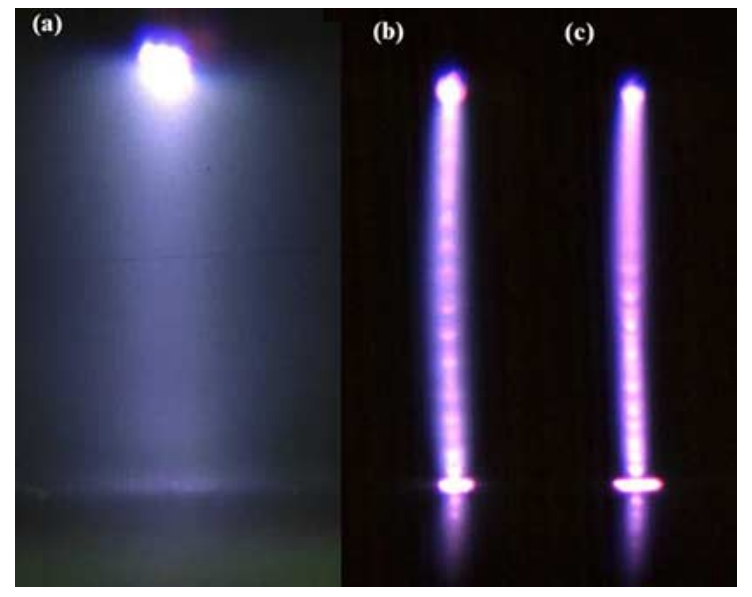

Fig. 2. Helium APGD formed between a top pin electrode and a bottom plate electrode in (a) homogenous mode, (b) striation mode, and (c) asymmetric striation mode.

the top electrode. It is worth noting that the less bright region near the top electrode appears to have a larger radius. Though not shown here, it is also possible to form the heavily striated region near the top electrode. At present, the conditions for the asymmetric pattern formation remains unclear but may relate to surface conditions of the electrodes.

Plasma striation can be observed in other electrode configurations. Fig. 2 shows images of helium APGD established between a top pin electrode and a bottom plate electrode. Again, the electrode separation distance is $8 \mathrm{~mm}$.

Fig. 2(a) shows diffuse and homogenous plasma at a very small peak current density of $1.6 \mathrm{~mA} / \mathrm{cm}^{2}$. For APGD in helium, this current level is likely to correspond to an operation regime between the Townsend mode and the normal glow mode [7]. Increasing the applied voltage further to an appropriate point, the homogenous APGD evolves suddenly into the symmetrically striated pattern of Fig. 2(b) at a peak discharge current density of $127 \mathrm{~mA} / \mathrm{cm}^{2}$. Similar to Fig. 1(e), the asymmetric striation pattern can also be sustained in this electrode configuration and an example is shown in Fig. 2(c).

Ion-wave has been considered as a mechanism for the establishment of double layers in space plasmas. It remains to confirm if it is responsible for the formation of multilayer plasma patterns in APGD. Yet it is clear that a very large local electric field is prerequisite for striation to be established. From a broader prospective, this observation suggests that once appropriate operation conditions are satisfied, a global self-adjustment in the spatial profile of the electric field can be facilitated by APGD itself to enable striation. Much needs to be studied before further insight into possible striation mechanisms can be substantiated.

\section{REFERENCES}

[1] D. W. Swain and S. C. Brown, "Moving striation in a low-pressure argon discharge," Phys. Fluids, vol. 14, pp. 1383-1393, July 1971.

[2] W. Breazeal, K. M. Flynn, and E. G. Gwinn, "Static and dynamic twodimensional patterns in self-extinguishing discharge avalanches," Phys. Rev. E, Stat. Phys. Plasmas Fluids Relat. Interdiscip. Top., vol. 52, pp. 1503-1515, 1995.

[3] K. H. Schoenbach, M. Moselhy, and W. Shi, "Self-organization in cathode boundary layer microdischarges," Plasma Sources Sci. Technol., vol. 13, pp. 177-185, Feb. 2004

[4] D. Diebold, C. E. Forest, N. Hershkowitz, M.-K. Hsieh, T. Intrator, D. Kaufman, G.-H. Kim, S.-G. Lee, and J. Menard, "Double-layer-relevant laboratory results," IEEE Trans. Plasma Sci., vol. 20, no. 6, pp. 601-606, Dec. 1992

[5] L. Conde and L. Leon, "Multiple double layers in a glow discharge," Phys. Plasma, vol. 1, pp. 2441-2447, Aug. 1994.

[6] Y. Yang, "Alternating-current glow and pseudoglow discharges in atmospheric pressure," IEEE Trans. Plasma Sci., vol. 31, no. 1, pp. 174-176, Feb. 2003.

[7] M. G. Kong and X. T. Deng, "Energy efficient production of a nonthermal atmospheric pressure gas discharge," IEEE Trans., Plasma Sci. vol. 31, no. 1, pp. 7-14, Feb. 2003. 